\title{
Effects of Socio-demographic Factors on the Decreasing Trend in the Sex Ratio at Birth in Korea, 1997-2017
}

\section{Jisuk Bae}

Department of Preventive Medicine, Daegu Catholic University School of Medicine, Daegu, Korea

Objectives: This study investigated the effects of socio-demographic factors on the decreasing trend in the sex ratio at birth from 1997 to 2017 in Korea.

Methods: Data from 10349602 live births registered with Statistics Korea from 1997 to 2017 were analyzed. The secondary sex ratio (SSR), defined as the ratio of male to female live births, during the study period (1997-1999 [phase I], 2000-2002 [phase II], 2003-2005 [phase III], 2006-2008 [phase IV], 2009-2011 [phase V], 2012-2014 [phase VI], and 2015-2017 [phase VII]) was calculated according to selected socio-demographic factors, such as parental age, education, occupation, and birth order. Logistic regression models were used to estimate odds ratios (ORs) and 95\% confidence intervals for a male birth after adjusting for potential confounders.

Results: The SSR gradually decreased from 1.10 (in 1998-2000 and 2002) to 1.05 (in 2013-2016). While a decreasing trend in the SSR was not noted among first births, male-biased sex ratios were prominent among third and higher-order births, for which the highest SSR was 1.46 in 1998. Higher birth order was significantly associated with an excess of male births in phases I-VI ( $\geq$ third vs. first, OR range, 1.03 to 1.35). Advanced maternal age was significantly associated with an excess of female births in phases II, III, and V ( $\geq 40$ vs. 20-24 years, OR range, 0.92 to 0.95 ).

Conclusions: This study demonstrated that advanced maternal age and reduction of the artificially-biased SSR among third and higher-order births may partially explain the decreasing trend in the SSR from 1997 to 2017 in Korea.

Key words: Birth order, Educational status, Maternal age, Occupations, Sex ratio, Socioeconomic factors

\section{INTRODUCTION}

The sex ratio at birth, or the secondary sex ratio (SSR), is defined as the ratio of male to female live births. Not only has the SSR served as a population health indicator (e.g., for fertility), but it has also played an important role as a social indicator

Received: June 16, 2020 Accepted: July 21, 2020

Corresponding author: Jisuk Bae, MD, PhD

Department of Preventive Medicine, Daegu Catholic University School of Medicine, 33 Duryugongwon-ro 17-gil, Nam-gu, Daegu 42472, Korea E-mail: jialove@cu.ac.kr

This is an Open Access article distributed under the terms of the Creative Commons Attribution Non-Commercial License (https://creativecommons.org/licenses/bync/4.0/) which permits unrestricted non-commercial use, distribution, and reproduction in any medium, provided the original work is properly cited. (e.g., for son preference, gender equity, and couples' childbearing behavior) in many countries around the world, notably in Asia [1-3]. Korea is an Asian country that has undergone drastic changes in the SSR in the last few decades [4]. In Korea, the SSR began to increase abruptly in the mid-1980s, along with the use of ultrasound for prenatal sex determination $[2,3,5]$. The SSR then peaked in 1990, with a severely male-biased SSR of 1.165. Since the mid-1990s, the SSR has gradually decreased, reaching an average natural SSR of 1.050 in 2016.

The stability and variability of the SSR have been proposed to be influenced by a variety of endogenous and exogenous factors. It has been suggested that several biological factors, such as parental hormone levels around the time of conception [6-8], follicular phase length $[9,10]$, timing of conception 
during the menstrual cycle $[7,9]$, and sperm characteristics [11], may play a role in the natural SSR. In addition, the SSR observed at the population level has been reported to vary according to socio-demographic factors such as parental age [12-15], birth order [12-14,16,17], race/ethnicity [12,14,18], and socioeconomic status (SES) $[5,12,15,19-22]$.

Despite the recent dramatic changes in the SSR observed in Korea, sparse previous research using nationally representative data has investigated the associations of socio-demographic factors with long-term temporal trends in the SSR. A study using national vital statistics data from 1981 to 2004 [5] assessed parental SES, as measured by education and occupation, in relation to the SSR, and suggested that maternal SES may be a more sensitive indicator of variations in the SSR than paternal SES. Of note, the aforementioned study reported that there had been changes in the association between parental SES and the SSR across three equidistant time periods (19811984, 1991-1994, and 2001-2004). Namely, before the mid1980s, higher parental SES was associated with an increased SSR (i.e., an excess of male births). Since then, however, an inverse association between parental SES and the SSR was prominently noted in the early 1990s. With a more extensive evaluation of socio-demographic factors (e.g., parental age, education, occupation, and birth order) in relation to the SSR, the present study aimed to investigate the impact of socio-demographic factors on the decreasing trend in the SSR from 1997 to 2017 in Korea.

\section{METHODS}

\section{Research Data}

Data from all live births registered with Statistics Korea from 1997 to 2017 were obtained from the Microdata Integrated Service (MDIS) [4]. With no missing values for infant sex, a total of 10349602 live births were included in the final dataset. Nonetheless, missing values for independent variables (birth order, $\mathrm{n}=44280$ [0.4\%]; maternal age, $\mathrm{n}=15519$ [0.1\%]; paternal age, $\mathrm{n}=82823$ [0.8\%]; maternal education, $\mathrm{n}=64135$ [0.6\%]; paternal education, $\mathrm{n}=104555$ [1.0\%]; maternal occupation, $\mathrm{n}=171102$ [1.7\%]; and paternal occupation, $\mathrm{n}=324730$ [3.1\%]) were excluded from the analysis of each variable.

\section{Statistical Analysis}

All live births, the proportions of male and female births and the SSR (among all live births and according to birth order [first, second, and third and higher-order births]) during the study period were calculated. The linear-by-linear association test was used to test the significance of trends in the proportions of male and female births during the study period. To evaluate changes in the SSR and its associations with sociodemographic factors over time, the study period was divided into 7 phases: 1997-1999 (phase l), 2000-2002 (phase II), 20032005 (phase III), 2006-2008 (phase IV), 2009-2011 (phase V), 2012-2014 (phase VI), and 2015-2017 (phase VII). Logistic regression models were used to estimate odds ratios (ORs) and 95\% confidence intervals ( $\mathrm{Cls}$ ) for a male birth, after adjusting for parental age ( $\leq 19,20-24,25-29,30-34,35-39$, and $\geq 40$ years), education (primary, secondary, tertiary), occupation (non-manual, manual, and others [students, housewives, etc.]), and/or birth order (first, second, and third or higher). As birth order has been recognized as a factor responsible for imbalances in the SSR, a subgroup analysis was conducted according to birth order. A 2-sided significance level of 0.05 was used to assess statistical significance. Statistical analysis was performed using SPSS version 25 (IBM Corp., Armonk, NY, USA).

\section{Ethics Statement}

As all of the data used were de-identified, this study was classified as exempt research by the Institutional Review Board of Daegu Catholic University Medical Center.

\section{RESULTS}

Table 1 shows all live births, the proportions of male and female births, and the SSR from 1997 to 2017 in Korea. During the study period, the highest SSR (1.10) was observed in the late 1990s and the early 2000s. The SSR gradually decreased from 1.10 (in 1998-2000 and 2002) to 1.05 (in 2013-2016). When the SSR was calculated according to birth order, the decreasing trend in the SSR was not noticeable among first births. Among third and higher-order births, however, malebiased sex ratios were prominent in the late 1990s and the early 2000s (with the highest SSR being 1.46 in 1998), demonstrating a remarkable decrease in the SSR during the study period (Table 1).

The number of all live births and the SSR according to sociodemographic factors in phases I-VII are presented in Table 2. The proportion of mothers aged $\geq 35$ years increased from $5.9 \%$ in phase I to $26.3 \%$ in phase VII; and that of fathers aged 


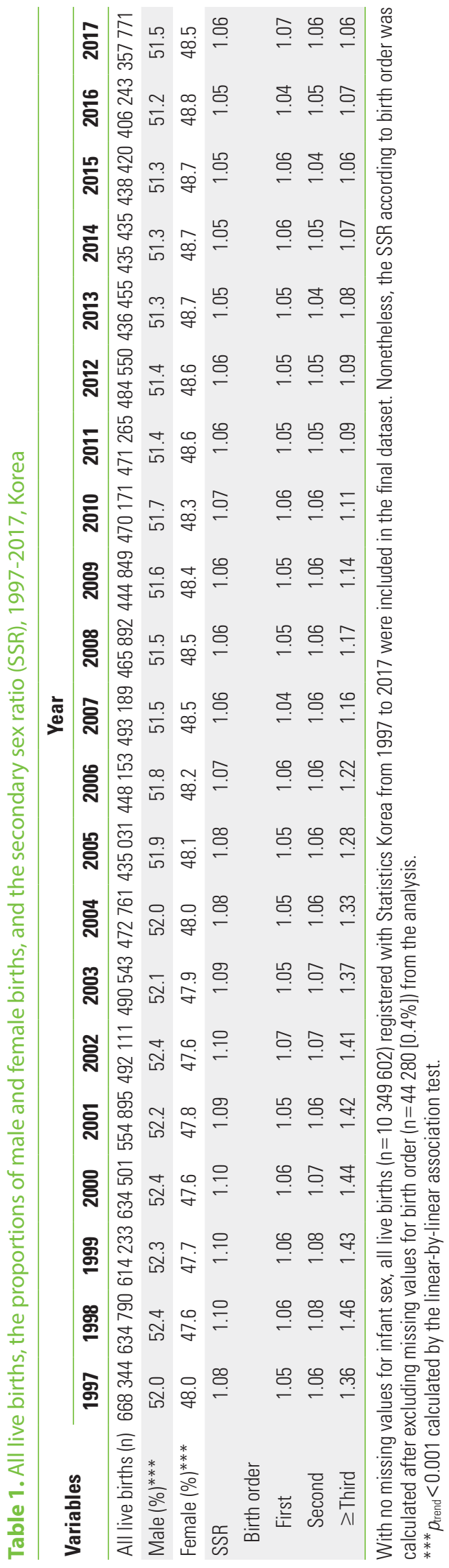

$\geq 35$ years increased from $17.8 \%$ in phase I to $47.2 \%$ in phase VII. The distributions of parental education and occupation changed markedly during the same period. For instance, the proportion of college-educated mothers increased from $32.0 \%$ to $77.2 \%$, and that of mothers with non-manual jobs increased from $10.1 \%$ to $32.4 \%$ (Table 2).

Table 3 presents the ORs and $95 \%$ Cls for a male birth according to socio-demographic factors among all live births. Higher birth order was significantly associated with an excess of male births in phases I-VI ( $\geq$ third vs. first, OR range, 1.03 to 1.35), but not in phase VII. Adolescent mothers had higher odds of a male birth than did mothers aged $20-24$ years in phase I $(\leq 19$ vs. $20-24$ years, $\mathrm{OR}, 1.04 ; 95 \% \mathrm{Cl}, 1.00$ to $1.07 ; p<0.05)$. However, older mothers had lower odds of a male birth than did mothers aged $20-24$ years in phases II, III, and V ( $\geq 40$ vs. $20-24$ years, OR range, 0.92 to 0.95 ). Equivocal findings on the association of maternal SES with the SSR were noted. Namely, less-educated mothers had lower odds of a male birth than did collegeeducated mothers in phase II (primary vs. tertiary, OR, 0.94; $95 \% \mathrm{Cl}, 0.90$ to 0.98 ). Meanwhile, compared with mothers with non-manual jobs, those with manual jobs $(\mathrm{OR}, 1.03 ; 95 \% \mathrm{Cl}$, 1.01 to 1.05$)$ and others $(\mathrm{OR}, 1.01 ; 95 \% \mathrm{Cl}, 1.00$ to $1.02 ; p<0.05)$ had higher odds of a male birth in phase III.

The associations between socio-demographic factors and the SSR among first births, second births, and third and higher-order births are shown in Tables 4 and 5, Supplemental Material 1. Comparable findings on the association between maternal age and the SSR were noted among first births (in phase I, $\leq 19$ vs. $20-24$ years, OR, $1.04 ; 95 \% \mathrm{Cl}, 1.01$ to 1.08 ; in phases $\mathrm{II}$, III, and V, $\geq 40$ vs. $20-24$ years, OR range, 0.87 to 0.93 ) relative to the corresponding findings among all live births. Paternal age was also found to be significantly associated with the SSR among first births, indicative of similar opposing directions toward infant sex at both age extremes (i.e., $\leq 19$ and $\geq 40$ years) (Table 4). However, when the analysis was restricted to third and higher-order births, the findings on the associations between parental age and the SSR were in contrast with the corresponding findings among all live births or first births, indicating that advanced parental age was associated with an excess of male births in phase I (Table 5). In addition, among third and higher-order births, fathers with low SES, as measured by education and occupation, had lower odds of a male birth than did those with high SES, particularly in some early phases (phases I-III) (Table 5). 


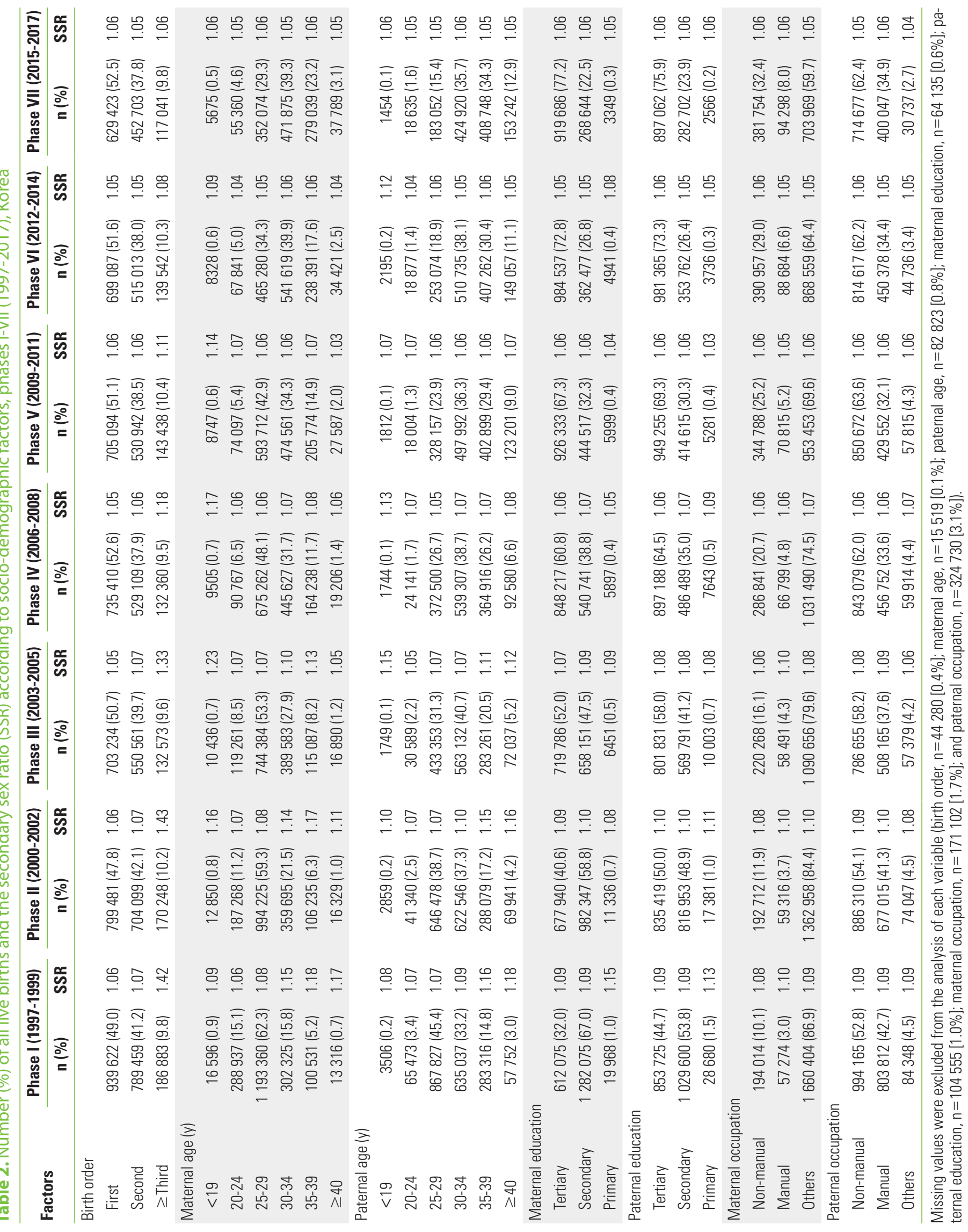


Table 3. Odds ratios (ORs) and 95\% confidence intervals (Cls) for a male birth according to socio-demographic factors among all live births, phases I-VII (1997-2017), Korea

\begin{tabular}{|c|c|c|c|c|c|c|c|}
\hline Variables & $\begin{array}{c}\text { Phase I } \\
(1997-1999)\end{array}$ & $\begin{array}{c}\text { Phase II } \\
(2000-2002)\end{array}$ & $\begin{array}{c}\text { Phase III } \\
(2003-2005)\end{array}$ & $\begin{array}{c}\text { Phase IV } \\
(2006-2008)\end{array}$ & $\begin{array}{c}\text { Phase V } \\
(2009-2011)\end{array}$ & $\begin{array}{c}\text { Phase VI } \\
(2012-2014)\end{array}$ & $\begin{array}{c}\text { Phase VII } \\
(2015-2017)\end{array}$ \\
\hline \multicolumn{8}{|l|}{ Birth order } \\
\hline First & 1.00 (reference) & 1.00 (reference) & 1.00 (reference) & 1.00 (reference) & 1.00 (reference) & 1.00 (reference) & 1.00 (reference) \\
\hline Second & $1.02(1.01,1.02)^{*}$ & $1.01(1.00,1.02)^{*}$ & $1.02(1.01,1.02)^{*}$ & $1.01(1.00,1.02)^{*}$ & $1.00(1.00,1.01)$ & $0.99(0.99,1.00)$ & $1.00(0.99,1.01)$ \\
\hline$\geq$ Third & $1.34(1.33,1.36)^{*}$ & $1.35(1.34,1.37)^{*}$ & $1.28(1.26,1.29)^{*}$ & $1.13(1.12,1.15)^{*}$ & $1.06(1.05,1.08)^{*}$ & $1.03(1.01,1.04)^{*}$ & $0.99(0.97,1.00)$ \\
\hline \multicolumn{8}{|l|}{ Maternal age (y) } \\
\hline$<19$ & $1.04(1.00,1.07)^{*}$ & $1.02(0.98,1.06)$ & $1.00(0.95,1.06)$ & $0.98(0.93,1.03)$ & $0.97(0.92,1.03)$ & $1.02(0.96,1.08)$ & $0.99(0.92,1.07)$ \\
\hline $20-24$ & 1.00 (reference) & 1.00 (reference) & 1.00 (reference) & 1.00 (reference) & 1.00 (reference) & 1.00 (reference) & 1.00 (reference) \\
\hline $25-29$ & $1.00(1.00,1.01)$ & $0.99(0.98,1.00)$ & $0.99(0.98,1.01)$ & $1.00(0.99,1.02)$ & $1.00(0.99,1.02)$ & $1.01(0.99,1.02)$ & $0.99(0.97,1.01)$ \\
\hline $30-34$ & $1.01(1.00,1.02)$ & $0.99(0.98,1.00)$ & $1.00(0.98,1.01)$ & $1.00(0.99,1.02)$ & $1.00(0.98,1.02)$ & $1.02(1.00,1.03)$ & $1.00(0.98,1.02)$ \\
\hline $35-39$ & $1.01(0.99,1.02)$ & $0.99(0.97,1.01)$ & $1.00(0.98,1.02)$ & $1.00(0.98,1.02)$ & $1.00(0.98,1.02)$ & $1.02(1.00,1.04)$ & $0.99(0.97,1.02)$ \\
\hline$\geq 40$ & $1.00(0.96,1.04)$ & $0.94(0.91,0.98)^{*}$ & $0.92(0.89,0.95)^{*}$ & $0.97(0.94,1.01)$ & $0.95(0.92,0.98)^{*}$ & $0.99(0.97,1.02)$ & $1.00(0.97,1.03)$ \\
\hline \multicolumn{8}{|l|}{ Paternal age (y) } \\
\hline$<19$ & $0.99(0.92,1.06)$ & $1.00(0.92,1.09)$ & $1.11(1.00,1.23)$ & $1.07(0.96,1.19)$ & $1.04(0.94,1.16)$ & $1.06(0.96,1.17)$ & $1.00(0.89,1.12)$ \\
\hline $20-24$ & 1.00 (reference) & 1.00 (reference) & 1.00 (reference) & 1.00 (reference) & 1.00 (reference) & 1.00 (reference) & 1.00 (reference) \\
\hline $25-29$ & $0.99(0.98,1.01)$ & $0.99(0.97,1.01)$ & $1.01(0.99,1.04)$ & $0.98(0.95,1.00)$ & $0.98(0.95,1.02)$ & $1.01(0.98,1.04)$ & $1.00(0.97,1.03)$ \\
\hline $30-34$ & $0.99(0.97,1.01)$ & $1.00(0.97,1.02)$ & $1.00(0.97,1.03)$ & $0.98(0.95,1.01)$ & $0.98(0.95,1.01)$ & $1.00(0.97,1.03)$ & $1.00(0.96,1.03)$ \\
\hline $35-39$ & $1.00(0.98,1.02)$ & $1.00(0.98,1.02)$ & $1.00(0.97,1.03)$ & $0.97(0.95,1.00)$ & $0.98(0.95,1.01)$ & $1.00(0.97,1.03)$ & $1.00(0.97,1.04)$ \\
\hline$\geq 40$ & $0.99(0.96,1.01)$ & $0.98(0.96,1.01)$ & $0.99(0.96,1.02)$ & $0.97(0.94,1.00)$ & $0.99(0.95,1.02)$ & $1.00(0.97,1.03)$ & $1.01(0.97,1.04)$ \\
\hline \multicolumn{8}{|c|}{ Maternal education } \\
\hline Tertiary & 1.00 (reference) & 1.00 (reference) & 1.00 (reference) & 1.00 (reference) & 1.00 (reference) & 1.00 (reference) & 1.00 (reference) \\
\hline Secondary & $1.00(0.99,1.01)$ & $1.00(1.00,1.01)$ & $1.00(0.99,1.00)$ & $1.00(0.99,1.00)$ & $1.00(0.99,1.01)$ & $1.00(0.99,1.01)$ & $1.00(0.99,1.01)$ \\
\hline Primary & $1.00(0.97,1.03)$ & $0.94(0.90,0.98)^{*}$ & $0.99(0.94,1.05)$ & $0.96(0.91,1.02)$ & $1.00(0.95,1.06)$ & $1.03(0.97,1.09)$ & $1.02(0.94,1.09)$ \\
\hline \multicolumn{8}{|c|}{ Paternal education } \\
\hline Tertiary & 1.00 (reference) & 1.00 (reference) & 1.00 (reference) & 1.00 (reference) & 1.00 (reference) & 1.00 (reference) & 1.00 (reference) \\
\hline Secondary & $0.99(0.98,1.00)^{*}$ & $0.99(0.98,1.00)^{*}$ & $0.99(0.99,1.00)$ & $1.00(0.99,1.01)$ & $0.99(0.98,1.00)^{*}$ & $1.00(0.99,1.01)$ & $1.00(0.99,1.01)$ \\
\hline Primary & $0.99(0.96,1.02)$ & $0.98(0.95,1.01)$ & $0.97(0.93,1.02)$ & $1.02(0.97,1.07)$ & $0.96(0.91,1.02)$ & $1.00(0.93,1.07)$ & $0.99(0.91,1.07)$ \\
\hline \multicolumn{8}{|c|}{ Maternal occupation } \\
\hline Non-manual & 1.00 (reference) & 1.00 (reference) & 1.00 (reference) & 1.00 (reference) & 1.00 (reference) & 1.00 (reference) & 1.00 (reference) \\
\hline Manual & $0.98(0.96,1.00)$ & $0.99(0.97,1.01)$ & $1.03(1.01,1.05)^{*}$ & $1.00(0.98,1.02)$ & $1.00(0.98,1.01)$ & $0.99(0.98,1.01)$ & $1.00(0.99,1.02)$ \\
\hline Others & $1.00(0.99,1.01)$ & $1.00(0.99,1.01)$ & $1.01(1.00,1.02)^{*}$ & $1.00(0.99,1.01)$ & $1.00(1.00,1.01)$ & $1.00(0.99,1.00)$ & $1.01(1.00,1.02)$ \\
\hline \multicolumn{8}{|c|}{ Paternal occupation } \\
\hline Non-manual & 1.00 (reference) & 1.00 (reference) & 1.00 (reference) & 1.00 (reference) & 1.00 (reference) & 1.00 (reference) & 1.00 (reference) \\
\hline Manual & $0.99(0.99,1.00)$ & $1.00(1.00,1.01)$ & $1.00(0.99,1.01)$ & $1.00(0.99,1.00)$ & $1.00(0.99,1.01)$ & $0.99(0.99,1.00)$ & $1.00(0.99,1.00)$ \\
\hline Others & $1.00(0.99,1.02)$ & $1.00(0.98,1.01)$ & $0.99(0.97,1.00)$ & $1.00(0.99,1.02)$ & $1.01(0.99,1.02)$ & $0.99(0.98,1.01)$ & $1.01(0.99,1.03)$ \\
\hline
\end{tabular}

Logistic regression models were used to estimate $\mathrm{ORs}$ and $95 \% \mathrm{Cls}$ for a male birth, after adjusting for birth order, parental age, education, and occupation. ${ }^{*} p<0.05$.

\section{DISCUSSION}

The present study used data from the national birth registry to evaluate the effects of selected socio-demographic factors on the decreasing trend in the SSR from 1997 to 2017 in Korea. This study revealed that higher birth order was a strong predictor of an excess of male births in 1997-2014, but not in 2015-
2017 (Table 3), possibly reflecting the reduction of an artificially-biased SSR among third and higher-order births over time [3]. In fact, the analysis according to birth order showed that changes in the SSR during the study period were not prominent among first births, but were noteworthy among third and higher-order births. Imbalances in the SSR observed in some Asian countries, such as China and India, are thought to 
Table 4. Odds ratios (ORs) and 95\% confidence intervals (Cls) for a male birth according to socio-demographic factors among first births, phases I-VII (1997-2017), Korea

\begin{tabular}{|c|c|c|c|c|c|c|c|}
\hline Variables & $\begin{array}{c}\text { Phase I } \\
(1997-1999)\end{array}$ & $\begin{array}{c}\text { Phase II } \\
(2000-2002)\end{array}$ & $\begin{array}{c}\text { Phase III } \\
(2003-2005)\end{array}$ & $\begin{array}{c}\text { Phase IV } \\
(2006-2008)\end{array}$ & $\begin{array}{c}\text { Phase V } \\
(2009-2011)\end{array}$ & $\begin{array}{c}\text { Phase VI } \\
(2012-2014)\end{array}$ & $\begin{array}{c}\text { Phase VII } \\
(2015-2017)\end{array}$ \\
\hline \multicolumn{8}{|c|}{ Maternal age (y) } \\
\hline$<19$ & $1.04(1.01,1.08)^{*}$ & $1.02(0.98,1.07)$ & $1.00(0.94,1.06)$ & $0.98(0.93,1.04)$ & $0.97(0.92,1.03)$ & $1.02(0.95,1.08)$ & $0.98(0.90,1.06)$ \\
\hline $20-24$ & 1.00 (reference) & 1.00 (reference) & 1.00 (reference) & 1.00 (reference) & 1.00 (reference) & 1.00 (reference) & 1.00 (reference) \\
\hline $25-29$ & $1.00(0.99,1.02)$ & $0.99(0.98,1.00)$ & $0.99(0.98,1.01)$ & $1.01(0.99,1.02)$ & $1.01(0.99,1.03)$ & $1.00(0.98,1.03)$ & $1.01(0.98,1.03)$ \\
\hline $30-34$ & $1.00(0.99,1.02)$ & $0.99(0.98,1.01)$ & $1.00(0.98,1.01)$ & $1.00(0.98,1.02)$ & $1.00(0.98,1.02)$ & $1.01(0.99,1.04)$ & $1.02(0.99,1.04)$ \\
\hline $35-39$ & $0.97(0.94,1.00)$ & $0.97(0.94,1.00)$ & $0.97(0.94,1.00)$ & $0.99(0.97,1.02)$ & $0.99(0.96,1.02)$ & $1.00(0.98,1.03)$ & $0.99(0.96,1.02)$ \\
\hline$\geq 40$ & $0.95(0.89,1.01)$ & $0.87(0.82,0.93)^{*}$ & $0.90(0.85,0.95)^{*}$ & $0.98(0.93,1.04)$ & $0.93(0.89,0.98)^{*}$ & $0.99(0.94,1.03)$ & $1.00(0.96,1.05)$ \\
\hline \multicolumn{8}{|l|}{ Paternal age (y) } \\
\hline$<19$ & $0.96(0.89,1.04)$ & $1.00(0.92,1.09)$ & $1.12(1.00,1.24)^{*}$ & $1.07(0.96,1.19)$ & $1.04(0.93,1.16)$ & $1.05(0.95,1.16)$ & $1.00(0.88,1.13)$ \\
\hline $20-24$ & 1.00 (reference) & 1.00 (reference) & 1.00 (reference) & 1.00 (reference) & 1.00 (reference) & 1.00 (reference) & 1.00 (reference) \\
\hline $25-29$ & $0.99(0.97,1.01)$ & $0.99(0.97,1.02)$ & $1.02(0.99,1.05)$ & $0.97(0.94,1.00)$ & $0.98(0.94,1.01)$ & $1.00(0.96,1.04)$ & $0.98(0.95,1.02)$ \\
\hline $30-34$ & $0.98(0.96,1.00)$ & $1.00(0.97,1.02)$ & $1.01(0.98,1.04)$ & $0.98(0.95,1.01)$ & $0.97(0.94,1.01)$ & $0.99(0.96,1.03)$ & $0.98(0.94,1.02)$ \\
\hline $35-39$ & $0.98(0.95,1.00)$ & $0.99(0.96,1.02)$ & $1.00(0.97,1.04)$ & $0.96(0.93,1.00)^{*}$ & $0.97(0.94,1.01)$ & $0.99(0.96,1.03)$ & $0.99(0.95,1.03)$ \\
\hline$\geq 40$ & $0.98(0.94,1.02)$ & $0.96(0.92,1.00)$ & $0.97(0.93,1.02)$ & $0.96(0.92,1.00)^{*}$ & $0.98(0.94,1.02)$ & $0.98(0.95,1.02)$ & $1.01(0.97,1.06)$ \\
\hline \multicolumn{8}{|c|}{ Maternal education } \\
\hline Tertiary & 1.00 (reference) & 1.00 (reference) & 1.00 (reference) & 1.00 (reference) & 1.00 (reference) & 1.00 (reference) & 1.00 (reference) \\
\hline Secondary & $0.99(0.98,1.01)$ & $1.00(0.99,1.01)$ & $0.99(0.98,1.01)$ & $1.00(0.99,1.01)$ & $0.99(0.98,1.01)$ & $1.00(0.99,1.02)$ & $1.01(0.99,1.02)$ \\
\hline Primary & $1.03(0.97,1.08)$ & $0.98(0.92,1.05)$ & $0.99(0.91,1.07)$ & $0.92(0.85,0.99)^{*}$ & $0.98(0.91,1.05)$ & $1.03(0.94,1.12)$ & $0.98(0.88,1.10)$ \\
\hline \multicolumn{8}{|c|}{ Paternal education } \\
\hline Tertiary & 1.00 (reference) & 1.00 (reference) & 1.00 (reference) & 1.00 (reference) & 1.00 (reference) & 1.00 (reference) & 1.00 (reference) \\
\hline Secondary & $1.00(0.99,1.01)$ & $1.00(0.99,1.01)$ & $1.00(0.99,1.01)$ & $1.01(1.00,1.02)$ & $0.99(0.98,1.00)$ & $0.99(0.98,1.01)$ & $0.99(0.98,1.01)$ \\
\hline Primary & $1.04(1.00,1.08)$ & $1.03(0.97,1.08)$ & $0.98(0.92,1.05)$ & $1.05(0.98,1.12)$ & $0.98(0.90,1.07)$ & $1.01(0.91,1.11)$ & $1.01(0.90,1.14)$ \\
\hline \multicolumn{8}{|c|}{ Maternal occupation } \\
\hline Non-manual & 1.00 (reference) & 1.00 (reference) & 1.00 (reference) & 1.00 (reference) & 1.00 (reference) & 1.00 (reference) & 1.00 (reference) \\
\hline Manual & $0.98(0.95,1.01)$ & $0.99(0.97,1.02)$ & $1.03(1.00,1.06)^{*}$ & $1.00(0.98,1.02)$ & $0.99(0.97,1.02)$ & $1.00(0.98,1.02)$ & $1.01(0.99,1.03)$ \\
\hline Others & $0.99(0.98,1.01)$ & $1.00(0.99,1.01)$ & $1.01(1.00,1.02)$ & $1.00(0.99,1.01)$ & $1.01(1.00,1.02)$ & $1.00(0.99,1.01)$ & $1.01(1.00,1.02)$ \\
\hline \multicolumn{8}{|c|}{ Paternal occupation } \\
\hline Non-manual & 1.00 (reference) & 1.00 (reference) & 1.00 (reference) & 1.00 (reference) & 1.00 (reference) & 1.00 (reference) & 1.00 (reference) \\
\hline Manual & $0.99(0.98,1.00)$ & $1.00(0.99,1.01)$ & $1.01(1.00,1.02)$ & $1.00(0.99,1.01)$ & $1.00(0.99,1.02)$ & $1.00(0.98,1.01)$ & $0.99(0.98,1.00)$ \\
\hline Others & $1.01(0.99,1.03)$ & $1.00(0.98,1.02)$ & $0.99(0.97,1.01)$ & $1.00(0.98,1.02)$ & $1.01(0.99,1.03)$ & $0.99(0.97,1.01)$ & $1.02(0.99,1.04)$ \\
\hline
\end{tabular}

Logistic regression models were used to estimate $\mathrm{ORs}$ and $95 \% \mathrm{Cls}$ for a male birth, after adjusting for parental age, education, and occupation. ${ }^{*} p<0.05$.

be attributed to son preference and prenatal sex selection against females $[2,3,5]$, which are social trends considered to be influenced by economics and cultural transmission [23,24]. As Korea has undergone drastic economic development and cultural changes during the last few decades, changes in sex selection practices, along with the implementation of policies against these practices, may have contributed to the rapid normalization of the artificially-biased SSR in Korea [24-26].

In addition, consistent with some-but not all-previous studies [12-15], the present study revealed that maternal age was significantly associated with differences in the SSR among all live births. Specifically, this study suggests that advanced maternal age ( $\geq 40$ years) may be associated with a lower SSR, whereas young maternal age ( $\leq 19$ years) may be associated with a higher SSR (Table 3). Similarly, in a study using data from the national birth registry from 1940 to 2002 in the United States, the 2 oldest maternal age groups ( $40-44$ and $\geq 45$ years) had the lowest SSR (both 1.04) [14]. Another study using United States linked birth-infant death data from 1983 to 2001 found that mothers aged 15-19 years were more likely to give birth to sons than mothers aged 20-34 years, whereas mothers aged $\geq 35$ years were more likely to give birth to daughters 
Table 5. Odds ratios (ORs) and $95 \%$ confidence intervals (Cls) for a male birth according to socio-demographic factors among third and higher-order births, phases I-VII (1997-2017), Korea

\begin{tabular}{|c|c|c|c|c|c|c|c|}
\hline Variables & $\begin{array}{c}\text { Phase I } \\
\text { (1997-1999) }\end{array}$ & $\begin{array}{c}\text { Phase II } \\
(2000-2002)\end{array}$ & $\begin{array}{c}\text { Phase III } \\
(2003-2005)\end{array}$ & $\begin{array}{l}\text { Phase IV } \\
(2006-2008)\end{array}$ & $\begin{array}{c}\text { Phase V } \\
(2009-2011)\end{array}$ & $\begin{array}{l}\text { Phase VI } \\
(2012-2014)\end{array}$ & $\begin{array}{l}\text { Phase VII } \\
(2015-2017)\end{array}$ \\
\hline \multicolumn{8}{|l|}{ Maternal age (y) } \\
\hline$<19$ & $0.53(0.31,0.91)^{*}$ & $0.54(0.29,1.00)$ & $1.18(0.57,2.42)$ & $1.93(0.35,10.61)$ & - & $0.72(0.28,1.86)$ & $0.94(0.39,2.27)$ \\
\hline $20-24$ & 1.00 (reference) & 1.00 (reference) & 1.00 (reference) & 1.00 (reference) & 1.00 (reference) & 1.00 (reference) & 1.00 (reference) \\
\hline $25-29$ & $1.14(1.07,1.21)^{*}$ & $1.04(0.97,1.11)$ & $1.05(0.96,1.15)$ & $0.97(0.87,1.08)$ & $0.95(0.85,1.07)$ & $0.97(0.86,1.08)$ & $0.96(0.85,1.09)$ \\
\hline $30-34$ & $1.24(1.16,1.32)^{*}$ & $1.10(1.02,1.18)^{*}$ & $1.11(1.01,1.22)^{*}$ & $1.02(0.92,1.14)$ & $1.02(0.91,1.13)$ & $0.98(0.87,1.09)$ & $0.97(0.86,1.10)$ \\
\hline $35-39$ & $1.25(1.17,1.34)^{*}$ & $1.09(1.01,1.17)^{*}$ & $1.11(1.01,1.22)^{*}$ & $1.02(0.92,1.14)$ & $1.01(0.91,1.14)$ & $0.99(0.89,1.11)$ & $0.96(0.85,1.09)$ \\
\hline$\geq 40$ & $1.29(1.18,1.41)^{*}$ & $1.06(0.97,1.16)$ & $1.00(0.90,1.11)$ & $0.95(0.84,1.07)$ & $0.99(0.88,1.11)$ & $0.98(0.87,1.11)$ & $0.94(0.83,1.07)$ \\
\hline \multicolumn{8}{|l|}{ Paternal age (y) } \\
\hline$<19$ & $0.74(0.07,8.38)$ & $3.78(0.41,34.61)$ & - & $3.23(0.33,31.39)$ & - & $1.83(0.42,7.98)$ & $0.47(0.08,2.68)$ \\
\hline $20-24$ & 1.00 (reference) & 1.00 (reference) & 1.00 (reference) & 1.00 (reference) & 1.00 (reference) & 1.00 (reference) & 1.00 (reference) \\
\hline $25-29$ & $1.26(1.06,1.49)^{*}$ & $0.94(0.76,1.17)$ & $0.92(0.74,1.15)$ & $1.25(0.99,1.58)$ & $1.05(0.83,1.35)$ & $1.10(0.87,1.39)$ & \\
\hline $30-34$ & $1.33(1.12,1.58)^{*}$ & $1.00(0.81,1.24)$ & $0.93(0.75,1.16)$ & $1.22(0.97,1.53)$ & $1.04(0.82,1.33)$ & $1.11(0.88,1.40)$ & $0.89(0.72,1.09)$ \\
\hline $35-39$ & $1.37(1.15,1.62)^{*}$ & $1.03(0.83,1.28)$ & $0.98(0.79,1.23)$ & $1.24(0.98,1.56)$ & $1.06(0.83,1.35)$ & $1.09(0.87,1.38)$ & $0.92(0.75,1.13)$ \\
\hline$\geq 40$ & $1.34(1.13,1.60)^{*}$ & $1.02(0.82,1.27)$ & $0.95(0.76,1.18)$ & $1.22(0.97,1.54)$ & $1.05(0.82,1.34)$ & $1.09(0.86,1.37)$ & $0.91(0.74,1.12)$ \\
\hline \multicolumn{8}{|c|}{ Maternal education } \\
\hline Tertiary & 1.00 (reference) & 1.00 (reference) & 1.00 (reference) & 1.00 (reference) & 1.00 (reference) & 1.00 (reference) & 1.00 (reference) \\
\hline Secondary & $1.04(1.01,1.07)^{*}$ & $1.03(1.00,1.06)^{*}$ & $1.04(1.01,1.07)^{*}$ & & & $0.99(0.97,1.02)$ & $0.99(0.97,1.02)$ \\
\hline Primary & $0.97(0.91,1.04)$ & $0.95(0.88,1.04)$ & $1.00(0.89,1.12)$ & $0.98(0.85,1.12)$ & $1.01(0.87,1.17)$ & $1.03(0.87,1.22)$ & $1.02(0.85,1.22)$ \\
\hline \multicolumn{8}{|c|}{ Paternal education } \\
\hline Tertiary & 1.00 (reference) & 1.00 (reference) & 1.00 (reference) & 1.00 (reference) & 1.00 (reference) & 1.00 (reference) & 1.00 (reference) \\
\hline Secondary & $0.95(0.93,0.98)^{*}$ & $0.94(0.92,0.97)^{*}$ & $0.91(0.89,0.94)^{*}$ & $0.96(0.94,0.99)^{*}$ & $0.98(0.95,1.00)$ & $0.99(0.96,1.01)$ & $1.01(0.98,1.04)$ \\
\hline Primary & $0.87(0.82,0.93)^{*}$ & $0.84(0.78,0.90)^{*}$ & $0.90(0.82,0.99)^{*}$ & $0.91(0.81,1.01)$ & $0.85(0.75,0.97)^{*}$ & $0.94(0.80,1.09)$ & $0.98(0.81,1.17)$ \\
\hline \multicolumn{8}{|c|}{ Maternal occupation } \\
\hline Non-manual & 1.00 (reference) & 1.00 (reference) & 1.00 (reference) & 1.00 (reference) & 1.00 (reference) & 1.00 (reference) & 1.00 (reference) \\
\hline Manual & $0.93(0.88,0.99)^{*}$ & $0.96(0.90,1.02)$ & $0.99(0.93,1.06)$ & $0.95(0.90,1.01)$ & $1.02(0.96,1.08)$ & $0.99(0.94,1.04)$ & $0.99(0.94,1.05)$ \\
\hline Others & $0.98(0.94,1.03)$ & $0.99(0.95,1.04)$ & $1.01(0.96,1.06)$ & $0.99(0.95,1.03)$ & $0.99(0.96,1.03)$ & $0.98(0.95,1.01)$ & $0.99(0.96,1.03)$ \\
\hline \multicolumn{8}{|c|}{ Paternal occupation } \\
\hline Non-manual & 1.00 (reference) & 1.00 (reference) & 1.00 (reference) & 1.00 (reference) & 1.00 (reference) & 1.00 (reference) & 1.00 (reference) \\
\hline Manual & $0.96(0.94,0.98)^{*}$ & $0.98(0.96,1.00)$ & $0.99(0.96,1.01)$ & $0.99(0.97,1.01)$ & $1.00(0.98,1.03)$ & $1.00(0.97,1.02)$ & $1.01(0.99,1.04)$ \\
\hline Others & $0.94(0.90,0.99)^{*}$ & $0.93(0.88,0.97)^{*}$ & $0.94(0.89,0.99)^{*}$ & $0.96(0.92,1.01)$ & $1.00(0.96,1.05)$ & $1.01(0.96,1.06)$ & $1.02(0.97,1.08)$ \\
\hline
\end{tabular}

Logistic regression models were used to estimate $\mathrm{ORs}$ and $95 \% \mathrm{Cls}$ for a male birth, after adjusting for parental age, education, and occupation. ${ }^{*} p<0.05$.

[15]. Contrary to the present study, however, several studies, particularly those conducted in Western countries, have suggested that higher birth order may be associated with a decreased SSR, given that older age at childbearing tends to be associated with higher birth order $[12,14]$. For instance, the aforementioned study conducted in the United States showed that seventh and eighth and higher-order births had the lowest SSR (both 1.03) [14].

Of note, the present study revealed that the association of parental age with the SSR differed by birth order. This may have resulted from a mixture of the biological and artificial effects of this factor on the SSR (i.e., a mixture of naturally-varied and artificially-biased SSR), which may differ by birth order. The SSR among first births, which appeared to be within the natural range of variability, was found to be significantly associated with parental age at both extremes ( $\leq 19$ and $\geq 40$ years), reflecting the potential biological impact of this factor on infant sex (Table 4). Meanwhile, the SSR for third and higher-order births, which appeared to be severely male-biased, particularly in the late 1990s (phase I), showed opposite results in terms of its association with parental age compared with the corresponding results among first births (Table 5). Among third and 
higher-order births in the late 1990s, as parental age increased, the odds of a male birth increased, implying possible birth cohort effects on the magnitude of son preference and couples' childbearing behavior [3,24,27].

Uncertain associations of parental SES with the SSR were noted among all live births and in the subgroup analysis according to birth order (Tables 3-5, and Supplemental Material 1). In comparison with a previous study using national vital statistics data from 1981 to 2004 [5], the present study did not clearly show that maternal SES was a more sensitive indicator of variation in the SSR than paternal SES during the study period. Instead, among third and higher-order births, low-SES fathers (SSR range, 1.21 to 1.32; data not shown) were found to have lower odds of a male birth than high-SES fathers (SSR range 1.37 to 1.49 ; data not shown), particularly in the late 1990s and early 2000s (phases I-III) (Table 5). This finding may be interpreted as reflecting the artificial effect of SES on the SSR, as high-SES fathers may have better access to sex selection techniques than low-SES fathers having a third and higher-order child.

In terms of the biological effect of SES on the SSR, the TriversWillard (TW) hypothesis, a long-established hypothesis regarding the SSR, theorizes that parents in good condition tend to produce more sons, whereas parents in poor condition tend to produce more daughters from the perspective of evolutionary biology [28]. According to the TW hypothesis, high-SES parents are more likely to have sons, while low-SES parents are more likely to have daughters, allegedly due to higher male mortality during the gestational period $[12,19,20,22]$. However, the existing evidence on the TW hypothesis is controversial, with inconsistent findings on the association between parental SES and the SSR $[12,15,19-22]$. For instance, in a study conducted in Sweden, more than 3000000 births between 1960 and 2007 were analyzed to examine various dimensions of parental SES (i.e., earnings, post-transfer income, wealth, parental wealth, education, and occupation) in relation to the SSR, and no substantive relationship was found between parental SES and the SSR [21]. In another study using Swedish national data for the years 1862 through 1991 [20], in contrast, economic contractions were found to be significantly associated with alterations in the SSR at the population level, implying a potential role of ambient social stressors such as economic stress in affecting the SSR $[19,20,22,29]$.

Nonetheless, the biological mechanisms by which parental socio-demographic factors might influence the SSR are ob- scure. Another prevailing hypothesis regarding the SSR is the hormonal hypothesis proposed by James [6-8], who postulated that parental hormone levels around the time of conception are potential determinants of offspring sex. According to this hypothesis, high levels of estrogen (in the mother) and testosterone (in either parent) are associated with an excess of male births, whereas high levels of follicle-stimulating hormone and luteinizing hormone are associated with an excess of female births [6,7]. In light of age-related hormonal changes in humans, parental age may function as a biological determinant of the SSR. While the hormonal hypothesis applies to the determination of sex ratio at conception or the primary sex ratio, the economic stress hypothesis proposed by Catalano applies to subsequent adjustments to the primary sex ratio via sex-selective fetal loss $[8,19,20]$. Namely, since maternal economic stress around the time of conception causes increased testosterone secretion from the adrenal glands, it may increase the likelihood of conceiving a male according to the hormonal hypothesis. If maternal economic stress persists during the gestational period, however, it may increase the risk of spontaneous abortion, which disproportionately affects male embryos and fetuses according to the economic stress hypothesis. As such, prolonged economic stress experienced by mothers may compensate for the male-biased primary sex ratio, or could even result in an excess of female births [29].

The major limitations of the present study should be taken into account when interpreting the observed results. Although the present study utilized national birth registry data that covered nearly all births in the nation, the information regarding the parents, such as age and SES, may have been inaccurate. As for the information on infants, the SSR is not considered to be affected by recall bias. With sole dependence on information included in the national birth registry, various dimensions of parental SES (e.g., earnings, wealth, etc.) were not evaluated. For the same reason, further investigations of other potential determinants of temporal trend in the SSR (e.g., environmental factors such as exposure to endocrine disruptors [30]) were unfeasible. Normative changes related to the observed trend in the SSR were not quantitatively assessed, meaning that this study did not address how much normative changes have contributed to the decreasing trend in the SSR during the study period. Furthermore, although the study results were interpreted as reflecting a mixture of the biological and artificial effects of factors affecting the SSR, the SSR may not be an accurate indicator for evaluating changes in sex selec- 
tion practices and policies targeting these practices within a population. As recently proposed, the sex selection propensity, which incorporates other relevant factors (e.g., family size and birth order) together with the SSR, may serve as a more accurate indicator of these changes [1].

In conclusion, the present study demonstrated that advanced maternal age and the reduction of the artificially-biased SSR among third and higher-order births may partially explain the decreasing trend in the SSR from 1997 to 2017 in Korea. The artificial effect of birth order on the SSR appeared to exceed the biological effect of birth order on the SSR from 1997 to 2014 , but this was no longer the case starting in the mid-2010s (2015-2017; phase VII). The rapid normalization of the artificially-biased SSR in Korea in the last few decades is perceived to be a desirable phenomenon, with favorable social implications regarding gender equity and couples' childbearing behavior. Along with various socio-demographic factors, potential environmental factors affecting the SSR at the population level need more research attention to ensure the stability of the SSR within its natural range of variability.

\section{SUPPLEMENTAL MATERIALS}

Supplemental material is available at https://doi.org/10.3961/ jpmph.20.282.

\section{CONFLICT OF INTEREST}

The author has no conflicts of interest associated with the material presented in this paper.

\section{FUNDING}

This work was supported by research grants from Daegu Catholic University in 2018.

\section{ACKNOWLEDGEMENTS}

None.

\section{AUTHOR CONTRIBUTIONS}

All work was done by JB.

\section{ORCID}

Jisuk Bae https://orcid.org/0000-0002-5809-5394

\section{REFERENCES}

1. Dubuc S, Sivia DS. Is sex ratio at birth an appropriate measure of prenatal sex selection? Findings of a theoretical model and its application to India. BMJ Glob Health 2018;3(4):e000675.

2. Hesketh T, Lu L, Xing ZW. The consequences of son preference and sex-selective abortion in China and other Asian countries. CMAJ 2011;183(12):1374-1377.

3. Choi EJ, Hwang J. Transition of son preference: evidence from South Korea. Demography 2020;57(2):627-652.

4. Statistics Korea. Annual report on live births and deaths statistics, 1997-2017 [cited 2020 Jun 2]. Available from: http:// kosis.kr/publication/publicationThema.do (Korean).

5. Chun H, Kim IH, Khang YH. Trends in sex ratio at birth according to parental social positions: results from vital statistics birth, 1981-2004 in Korea. J Prev Med Public Health 2009;42(2):143150 (Korean).

6. James WH. Evidence that mammalian sex ratios at birth are partially controlled by parental hormone levels around the time of conception. J Endocrinol 2008;198(1):3-15.

7. James WH. The variations of human sex ratio at birth with time of conception within the cycle, coital rate around the time of conception, duration of time taken to achieve conception, and duration of gestation: a synthesis. J Theor Biol 2008;255(2): 199-204.

8. James WH. Hypotheses on the stability and variation of human sex ratios at birth. J Theor Biol 2012;310:183-186.

9. Martin JF. Length of the follicular phase, time of insemination, coital rate and the sex of offspring. Hum Reprod 1997;12(3): 611-616.

10. Weinberg CR, Baird DD, Wilcox AJ. The sex of the baby may be related to the length of the follicular phase in the conception cycle. Hum Reprod 1995;10(2):304-307.

11. Bae J, Kim S, Chen Z, Eisenberg ML, Buck Louis GM. Human semen quality and the secondary sex ratio. Asian J Androl 2017; 19(3):374-381.

12. Chahnazarian A. Determinants of the sex ratio at birth: review of recent literature. Soc Biol 1988;35(3-4):214-235.

13. Jacobsen $\mathrm{R}$, Møller $\mathrm{H}$, Mouritsen A. Natural variation in the human sex ratio. Hum Reprod 1999;14(12):3120-3125.

14. Mathews TJ, Hamilton BE. Trend analysis of the sex ratio at 
birth in the United States. Natl Vital Stat Rep 2005;53(20):1-17.

15. Almond D, Edlund L. Trivers-Willard at birth and one year: evidence from US natality data 1983-2001. Proc Biol Sci 2007; 274(1624):2491-2496.

16. Biggar RJ, Wohlfahrt J, Westergaard T, Melbye M. Sex ratios, family size, and birth order. Am J Epidemiol 1999;150(9):957962.

17. Jiang Q, Yu Q, Yang S, Sánchez-Barricarte JJ. Changes in sex ratio at birth in China: a decomposition by birth order. J Biosoc Sci 2017;49(6):826-841.

18. Davis DL, Webster P, Stainthorpe H, Chilton J, Jones L, Doi R. Declines in sex ratio at birth and fetal deaths in Japan, and in U.S. whites but not African Americans. Environ Health Perspect 2007;115(6):941-946.

19. Catalano RA. Sex ratios in the two Germanies: a test of the economic stress hypothesis. Hum Reprod 2003;18(9):19721975.

20. Catalano RA, Bruckner T. Economic antecedents of the Swedish sex ratio. Soc Sci Med 2005;60(3):537-543.

21. Kolk M, Schnettler S. Socioeconomic status and sex ratios at birth in Sweden: no evidence for a Trivers-Willard effect for a wide range of status indicators. Am J Hum Biol 2016;28(1):6773.

22. Grech V. A socio-economic hypothesis for lower birth sex ratios at racial, national and global levels. Early Hum Dev 2018; 116:81-83.
23. Lipatov M, Li S, Feldman MW. Economics, cultural transmission, and the dynamics of the sex ratio at birth in China. Proc Natl Acad Sci U S A 2008;105(49):19171-19176.

24. Chung W, Gupta MD. The decline of son preference in South Korea: the roles of development and public policy. Popul Dev Rev 2007;33(4):757-783.

25. Korea Institute of Health and Social Affairs. A study of fertility changes in Korea: based on National Fertility and Family Health Survey (1974-2012). Sejong: Korea Institute of Health and Social Affairs; 2016, p. 158-203 (Korean).

26. Korea Institute of Health and Social Affairs. The 2015 National Survey on Fertility and Family Health and Welfare. Sejong: Korea Institute of Health and Social Affairs; 2015, p. 99-178 (Korean).

27. Lin TC. The decline of son preference and rise of gender indifference in Taiwan since 1990. Demogr Res 2009;20:377-402.

28. Trivers $\mathrm{RL}$, Willard DE. Natural selection of parental ability to vary the sex ratio of offspring. Science 1973;179(4068):90-92.

29. Bae J, Lynch CD, Kim S, Sundaram R, Sapra KJ, Buck Louis GM. Preconception stress and the secondary sex ratio in a population-based preconception cohort. Fertil Steril 2017;107(3): 714-722.

30. Bae J, Kim S, Barr DB, Buck Louis GM. Maternal and paternal serum concentrations of persistent organic pollutants and the secondary sex ratio: a population-based preconception cohort study. Environ Res 2018;161:9-16. 